\title{
Upregulation of COL8A1 indicates poor prognosis across human cancer types and promotes the proliferation of gastric cancer cells
}

\author{
JUN ZHOU, YANING SONG, WEI GAN, LIYE LIU, GUIBING CHEN, ZHENYU CHEN, \\ GUODE LUO, LIN ZHANG, GUOHU ZHANG, PEIHONG WANG and YONGKUAN CAO \\ Department of Gastrointestinal Surgery, The General Hospital of Western Theater Command, \\ Chengdu, Sichuan 610083, P.R. China
}

Received May 15, 2019; Accepted January 13, 2020

DOI: 10.3892/ol.2020.11895

\begin{abstract}
Gastric cancer (GC) was one of the most common types of the digestive system. COL8A1 was reported to be associated with cancer progression. The present study showed COL8A1 was overexpressed and correlated to shorter overall survival (OS) time across human cancer types. Specially, our results showed COL8A1 was up-regulated in advanced stage GC compared to low stage GC samples. Higher expression of COL8A1 was significantly correlated to shorter OS time in patients with GC. Bioinformatics analysis revealed COL8A1 was involved in regulating cell proliferation and metastasis. Experimental validations of COL8A1 showed that silencing of COL8A1 could significantly suppressed cell proliferation, migration and invasion in GC. These results provided a potential target for the clinical prognosis and treatment of gastric cancer.
\end{abstract}

\section{Introduction}

Cancer is a complex and intrinsically heterogeneous disease (1). An increased understanding of the mechanisms underlying the progression of human cancer may aid the identification of novel therapeutic targets. Next generation sequencing methods have enabled the development of a series of public databases, including The Cancer Genome Atlas (TCGA) (2) and the Gene Expression Profiling Interactive Analysis (GEPIA) (3) and Genotype-Tissue Expression databases (4), which may be used to investigate the heterogeneity and complexity of cancer. Gastric cancer (GC) is one of the most common tumors of the digestive system. The prognosis of patients with locally advanced GC is poor, and the 5-year survival rate is $<50 \%$ (5). In the past decades, studies have focused on identifying novel diagnostic and prognostic biomarkers for several types of

Correspondence to: Dr Yongkuan Cao, Department of Gastrointestinal Surgery, The General Hospital of Western Theater Command, 270 Tianhui Road, Rongdu Avenue, Chengdu, Sichuan 610083, P.R. China

E-mail: caoyok@163.com

Key words: prostate cancer, COL8A1, prognosis, proliferation, cell apoptosis human cancer, including GC. For example, TIMP metallopeptidase inhibitor 2 (TIMP2) was identified as a prognostic marker for GC, and increased expression of TIMP2 was correlated with a shorter survival time in patients with GC (6). However, effective biomarkers to improve the survival time of patients with $\mathrm{GC}$ are still required.

Collagen type VIII $\alpha 1$ chain (COL8A1) encodes one of the two $\alpha$ chains of collagen type VIII (7), which had been revealed to play a role in atherogenesis and vascular remodeling, presumably through modification of cell migration and proliferation $(8,9)$. COL8A1 is produced in the cornea, aortic endothelial cells and intraglomerular mesangial cells $(10,11)$. COL8A1 was found to promote the growth of smooth muscle cells, suggesting that it serves important roles in regulating cell biology (7). Moreover, previous studies have revealed that COL8A1 may be implicated in cancer progression. Di et al (12) used weighted gene co-expression network analysis to report that COL8A1 was an important stage-associated gene in bladder cancer. Shang et al (13) reported that COL8A1 was associated with the development and diagnosis of colon cancer. Furthermore, the knockdown of COL8A1 suppressed cell growth and invasion of hepatocarcinoma cells (14). However, despite the aforementioned studies, the expression pattern and molecular functions of COL8A1 in human cancer remain largely unclear.

The present study evaluated the mRNA levels of COL8A1 across different human cancer types and investigated the association between COL8A1 expression and survival time using TCGA database. Integrated analysis revealed that COL8A1 was upregulated across human cancer types, including GC. Bioinformatics analysis showed that COL8A1 was involved in regulating the cell cycle and DNA replication. Furthermore, increased expression of COL8A1 was associated with advanced stage and poor overall survival (OS) time in patients with GC. Additionally, silencing of COL8A1 significantly suppressed the proliferation and migration of GC cells in vitro. To the best of our knowledge, the present study is the first to demonstrate that COL8A1 may serve as a potential biomarker for GC.

\section{Materials and methods}

Public database analysis. COL8A1 expression data in Adrenocortical carcinoma (ACC), Bladder Urothelial 
Carcinoma (BLCA), Breast invasive carcinoma (BRCA), Cervical squamous cell carcinoma and endocervical adenocarcinoma (CESC), Cholangio carcinoma (CHOL), Colon adenocarcinoma (COAD), Lymphoid Neoplasm Diffuse Large B-cell Lymphoma (DLBC), Esophageal carcinoma (ESCA), Glioblastoma multiforme (GBM), Head and Neck squamous cell carcinoma (HNSC), Kidney Chromophobe (KICH), Kidney renal clear cell carcinoma (KIRC), Kidney renal papillary cell carcinoma (KIRP), Acute Myeloid Leukemia (LAML), Brain Lower Grade Glioma (LGG), Liver hepatocellular carcinoma (LIHC), Lung adenocarcinoma (LUAD), Lung squamous cell carcinoma (LUSC), Mesothelioma (MESO), Ovarian serous cystadenocarcinoma (OV), Pancreatic adenocarcinoma (PAAD), Pheochromocytoma and Paraganglioma (PCPG), Prostate adenocarcinoma (PRAD), Rectum adenocarcinoma (READ), Sarcoma (SARC), Skin Cutaneous Melanoma (SKCM), Stomach adenocarcinoma (STAD), Testicular Germ Cell Tumors (TGCT), Thyroid carcinoma (THCA), Thymoma (THYM), Uterine Corpus Endometrial Carcinoma (UCEC), Uterine Carcinosarcoma (UCS), Uveal Melanoma (UVM) datasets were downloaded from the GEPIA database (gepia.cancer-pku.cn/detail.php) on April 28, 2019.

The GSE15459 (15), GSE29272 (16), GSE51105 (17), GSE62254 (18), GSE15459 (19) and GSE14210 (20) datasets and the Kaplan-Meier Plotter database (21) were analyzed to determine the association between COL8A1 expression and overall survival time in patients with GC.

Cell culture. The human GC cell line AGS was purchased from the American Type Culture Collection and cultured in Dulbecco's modified Eagle's medium (Gibco; Thermo Fisher Scientific, Inc.) supplemented with $10 \%$ fetal bovine serum (Gibco; Thermo Fisher Scientific, Inc.)

Lentivirus transfection. The short hairpin (sh)RNA targeting human COL8A1 (5'-TGTATAACGGCAGACAGAA-3') and the negative control (NC) shRNA (5'-TTCTCCGAACGTGTC ACGT-3') were designed and inserted into the pGCSIL-GFP vector. The recombinant lentivirus was purchased from Shanghai GeneChem Co., Ltd. Stable knockdown of COL8A1 was achieved by transfecting the AGS cells with the lentiviral vector for $72 \mathrm{~h}$.

Reverse-transcription quantitative PCR (RT-qPCR). RT-qPCR was performed as previously described $(22,23)$. The primer sequences used for qPCR were as follows: COL8A1 forward, 5'-AGAACTACAACCCGCAGAC-3' and reverse, 5'-TTGAAT AGAGCAACCCACA-3'; and GAPDH forward, 5'-GGGAGC CAAAAGGGTCAT-3' and reverse, 5'-GAGTCCTTCCAC GATACCAA-3'. COL8A1 mRNA levels were quantified using the $2^{-\Delta \Delta \mathrm{Cq}}$ method (24) and normalized to the internal reference gene GAPDH.

Cell proliferation assay. Cell proliferation was assessed using the adherent cell cytometry system Celigo ${ }^{\circledR}$ and analyzed using Application Programing Interface (version 1.0; software). Briefly, 2,000 AGS cells transfected with shNC or shCOL8A1 were seeded in a 6 -well plate. The number of cells was counted after 1, 2, 3, 4 or 5 days. The experiment was performed in triplicate.
Table I. COL8A1 expression is upregulated in multiple types of human cancer.

\begin{tabular}{|c|c|c|c|}
\hline Cancer & $\begin{array}{l}\text { Median } \\
\text { (tumor) }\end{array}$ & $\begin{array}{l}\text { Median } \\
\text { (normal) }\end{array}$ & P-value \\
\hline $\mathrm{ACC}$ & 1.07 & 5.01 & $3.89 \times 10^{-16}$ \\
\hline BLCA & 5.14 & 8.19 & $5.82 \times 10^{-1}$ \\
\hline BRCA & 20.75 & 5.39 & $1.00 \times 10^{-34}$ \\
\hline CESC & 2.23 & 2.72 & $9.61 \times 10^{-1}$ \\
\hline CHOL & 5.86 & 0.32 & $8.37 \times 10^{-1}$ \\
\hline COAD & 7.54 & 5.87 & $3.85 \times 10^{-4}$ \\
\hline DLBC & 0.23 & 0.04 & $2.25 \times 10^{-16}$ \\
\hline ESCA & 7.82 & 2.41 & $2.01 \times 10^{-19}$ \\
\hline GBM & 5.19 & 0.19 & $2.23 \times 10^{-33}$ \\
\hline HNSC & 4.62 & 0.83 & $6.43 \times 10^{-9}$ \\
\hline $\mathrm{KICH}$ & 1.61 & 2.87 & $2.23 \times 10^{-1}$ \\
\hline KIRC & 23.36 & 4.06 & $2.43 \times 10^{-35}$ \\
\hline KIRP & 2.76 & 3.77 & $3.75 \times 10^{-1}$ \\
\hline LAML & 0.06 & 0.06 & $9.95 \times 10^{-1}$ \\
\hline LGG & 0.32 & 0.19 & $2.00 \times 10^{-10}$ \\
\hline LIHC & 0.76 & 0.25 & $1.80 \times 10^{-10}$ \\
\hline LUAD & 27.01 & 31.21 & $1.65 \times 10^{-5}$ \\
\hline LUSC & 13.43 & 31.81 & $1.39 \times 10^{-37}$ \\
\hline OV & 6.09 & 8.06 & $8.78 \times 10^{-1}$ \\
\hline PAAD & 25.99 & 0.34 & $3.26 \times 10^{-68}$ \\
\hline PCPG & 4.44 & 3.48 & $9.12 \times 10^{-1}$ \\
\hline PRAD & 7.28 & 8.78 & $7.99 \times 10^{-1}$ \\
\hline READ & 8.91 & 6.37 & $1.62 \times 10^{-3}$ \\
\hline SARC & 14.34 & 3.93 & $1.35 \times 10^{-1}$ \\
\hline SKCM & 1.78 & 1.82 & $4.49 \times 10^{-5}$ \\
\hline STAD & 13.43 & 1.48 & $4.93 \times 10^{-36}$ \\
\hline TGCT & 1.25 & 0.57 & $3.49 \times 10^{-5}$ \\
\hline THCA & 68.71 & 24.8 & $8.99 \times 10^{-49}$ \\
\hline THYM & 0.35 & 0.04 & $6.07 \times 10^{-12}$ \\
\hline UCEC & 1.70 & 3.29 & $5.71 \times 10^{-2}$ \\
\hline UCS & 2.64 & 3.29 & $3.71 \times 10^{-1}$ \\
\hline
\end{tabular}

Cell apoptosis. Flow cytometry was used to assess cell apoptosis. Briefly, $5 \times 10^{5}$ AGS cells transfected with shNC and shCOL8A1 were seeded into a 6-well plate and incubated for $48 \mathrm{~h}$. The cells were subsequently collected and washed twice with PBS. Apoptosis was detected using a flow cytometer and an Annexin V-APC Apoptosis Detection kit (eBioscience; Thermo Fisher Scientific, Inc.).

Bioinformatics analysis. The Database for Annotation, Visualization and Integrated Discovery (DAVID; david. ncifcrf.gov/home.jsp) was used for bioinformatics analysis.

Western blotting. Western blotting was performed as previously described (25). The primary antibodies used in the present study included anti-COL8A1 (cat. no. PA5-62731; Thermo Fisher Scientific, Inc.) and anti-GAPDH (cat. no. sc-32233; Santa-Cruz Biotechnology, Inc.). A goat anti-mouse secondary 

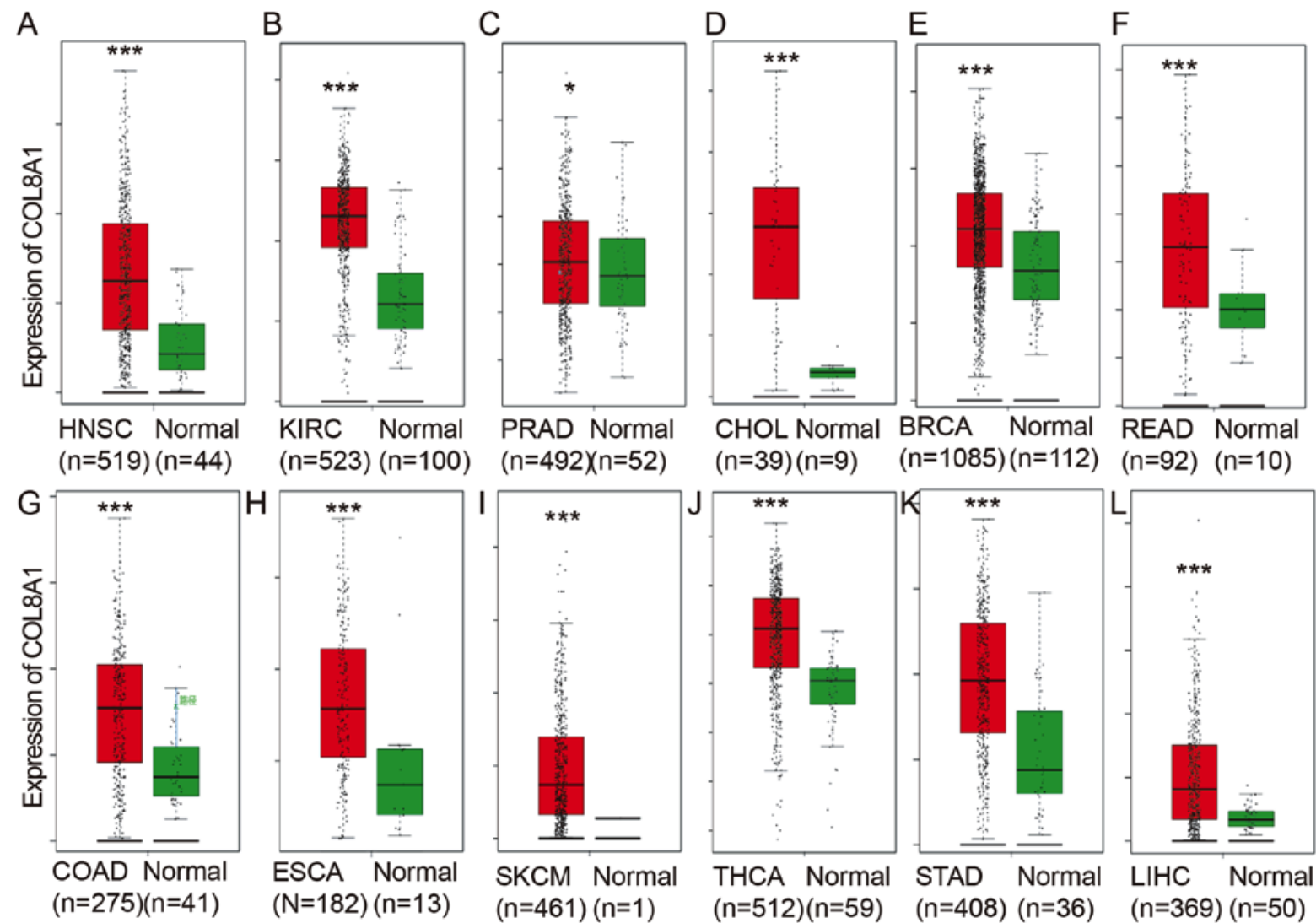

Figure 1. COL8A1 expression is upregulated across human cancer types. The present study analyzed the mRNA levels of COL8A1 in tumor tissues and normal samples across human cancer types, including (A) HNSC, (B) KIRC, (C) PRAD, (D) CHOL, (E) BRCA, (F) READ, (G) COAD, (H) ESCA, (I) SKCM, (J) THCA, (K) STAD and (L) LIHC. P $<0.05$ was considered to indicate a statistically significant difference. ${ }^{*} \mathrm{P}<0.05$; ${ }^{* * * *} \mathrm{P}<0.001$. TCGA, The Cancer Genome Atlas.

antibody (cat. no. sc-2005; Santa-Cruz Biotechnology, Inc.) was used.

Statistical analysis. Statistical analyses were performed using SPSS software (version 15.0; SPSS Inc.). Each experiment was performed in triplicate. The data are expressed as the mean \pm standard deviation. The Student's t-test or Mann-Whitney U-test was used to determine the association between COL8A1 expression and the clinical characteristics of the tumors. Kaplan-Meier analysis followed by the log-rank test and Cox regression analysis were used to assess the association between COL8A1 expression and the OS time of patients. $\mathrm{P}<0.05$ was considered to indicate a statistically significant difference.

\section{Results}

COL8A1 is upregulated across different types of human cancer. The mRNA levels of COL8A1 in tumor and normal tissues across human cancer types, including ACC, BLCA, BRCA, CESC, CHOL, COAD, DLBC, ESCA, GBM, HNSC, KICH, KIRC, KIRP, LAML, LGG, LIHC, LUAD, LUSC, MESO, OV, PAAD, PCPG, PRAD, READ, SARC, SKCM, STAD, TGCT, THCA, THYM, UCEC, UCS, and UVM, were analyzed using TCGA datasets. The results revealed that COL8A1 was significantly upregulated in HNSC, KIRC, PRAD, CHOL, CESC, BRCA, READ, LUSC, COAD, ESCA,
SKCM, BLCA, THCA, STAD and LIHC tissues compared with normal tissues (Fig. 1 and Table I), suggesting that COL8A1 may serve as an oncogene.

COL8A1 has prognostic value across human cancer types. The prognostic value of COL8A1 expression in human cancer was evaluated using Kaplan-Meier analysis and the log-rank test. Upregulated COL8A1 mRNA expression was significantly associated with OS time in several cancer types, including PAAD, OV, BLCA, BRCA, CESC, KIRC, LUSC and STAD (Fig. 2). Patients with high COL8A1 expression had a significantly shorter OS time compared with those with low expression. The results of these analyses, together with previous reports investigating bladder and colon cancer, suggested that COL8A1 may serve as a biomarker for cancer prognosis.

COL8A1 is upregulated in advanced GC samples. The present study revealed that COL8A1 was significantly upregulated in stage II-IV STAD compared with stage I STAD samples, in stage III and IV OV compared with stage II OV samples, in stage II and III ESCA compared with stage I ESCA samples and in stage III and IV OV compared with stage II BLCA samples (Fig. 3).

Upregulation of COL8A1 predicts a poor prognosis in GC. The present study revealed that high COL8A1 expression was associated with a shorter OS time in patients with GC. 

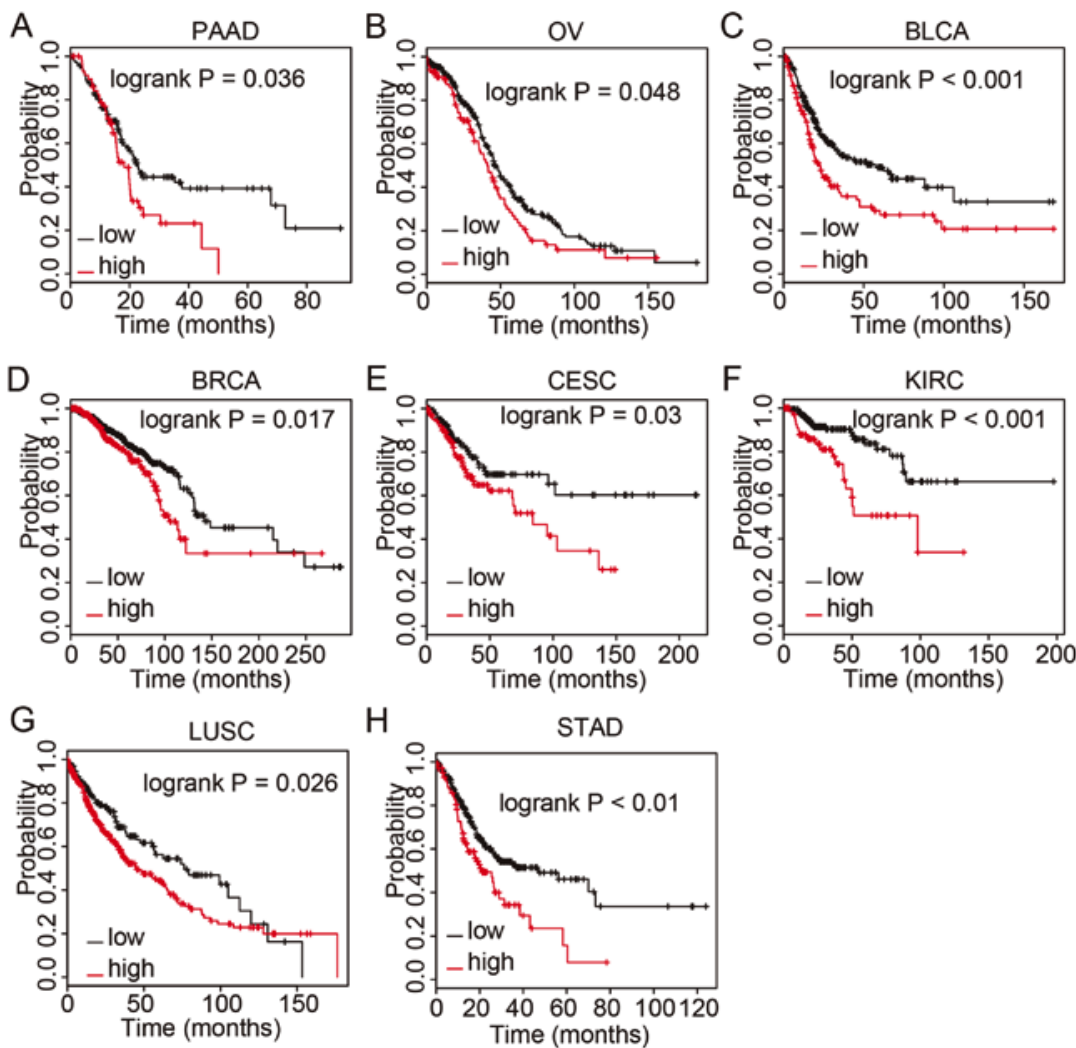

Figure 2. Higher COL8A1 expression is associated with shorter overall survival time across human cancer types. Elevation of COL8A1 mRNA expression was significantly associated with overall survival time in multiple cancer types, including (A) PAAD, (B) OV, (C) BLCA, (D) BRCA, (E) CESC, (F) KIRC, (G) LUSC and (H) STAD. P $<0.05$ was considered to indicate a statistically significant difference.

A

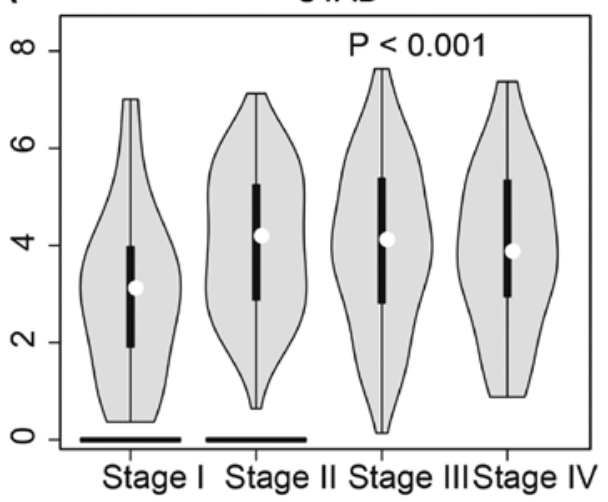

C

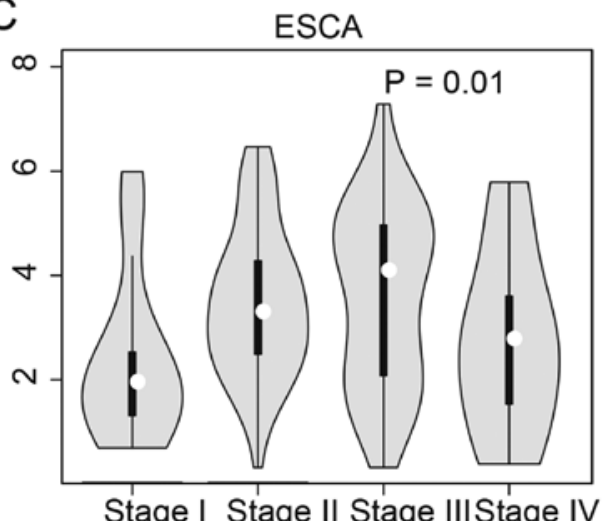

B
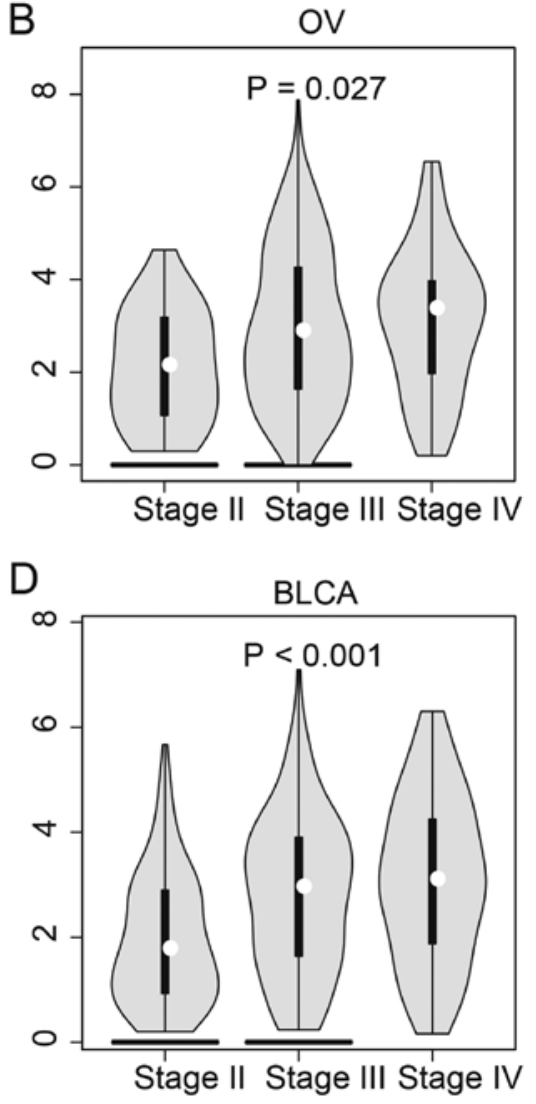

Figure 3. COL8A1 expression is upregulated in advanced stage STAD compared with low stage STAD samples. COL8A1 expression was upregulated in advanced stage (A) STAD, (B) OV, (C) ESCA and (D) BLCA compared with low stage cancer samples. P $<0.05$ was considered to indicate a statistically significant difference. STAD, gastric cancer; OV, ovarian serous cystadenocarcinoma; ESCA, esophageal carcinoma; BLCA, bladder urothelial carcinoma. 

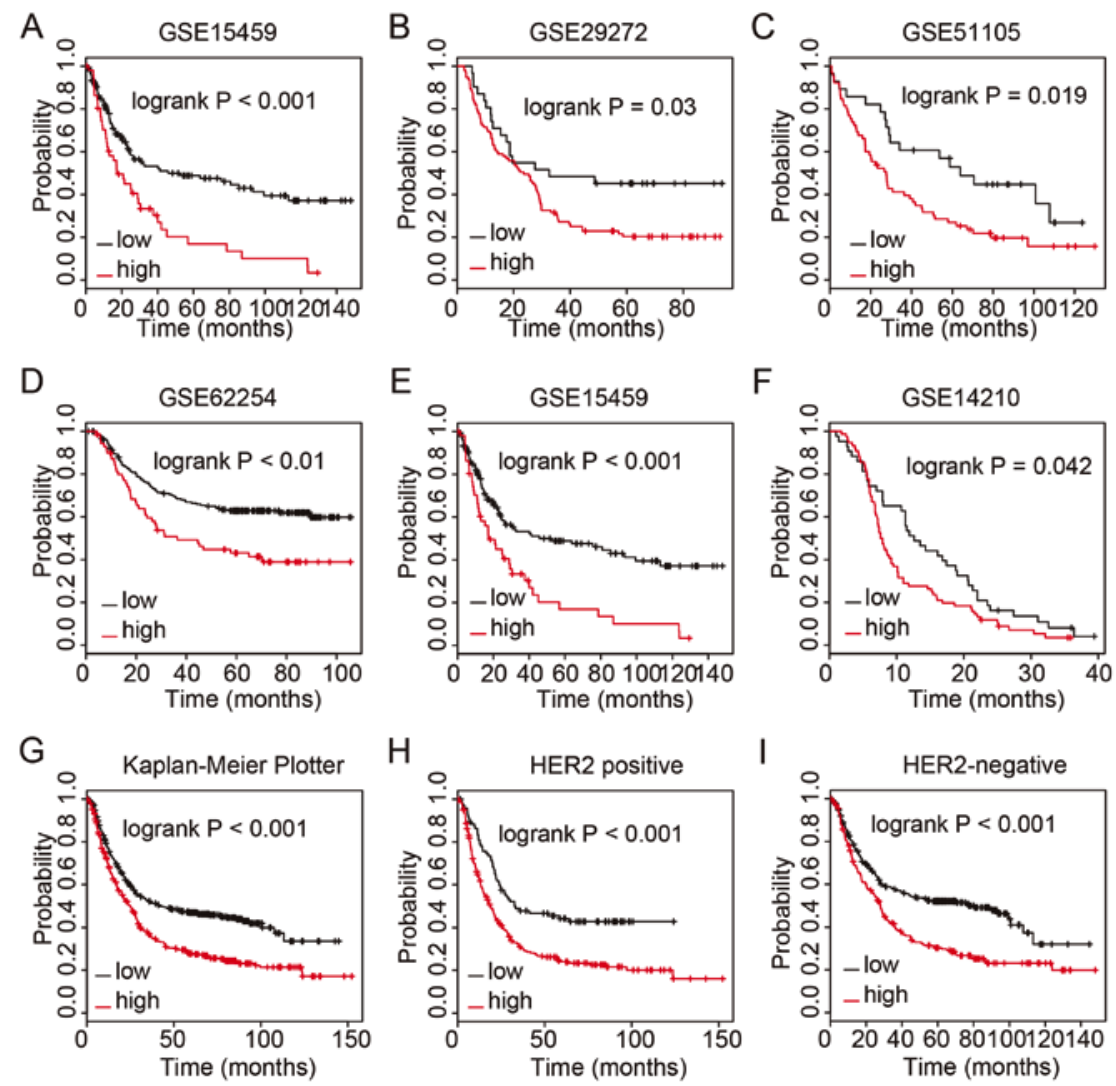

Figure 4. Higher COL8A1 expression is associated with shorter OS time in patients with GC. Higher COL8A1 expression was associated with shorter OS time in patients with GC in several datasets, including (A) GSE15459, (B) GSE29272, (C) GSE51105, (D) GSE62254, (E) GSE15459, (F) GSE14210 and (G) the Kaplan-Meier Plotter database. Higher expression of COL8A1 was associated with shorter OS time in both (H) HER2 positive and (I) negative GC samples. $\mathrm{P}<0.05$ was considered to indicate a statistically significant difference. OS, overall survival; GC, gastric cancer.
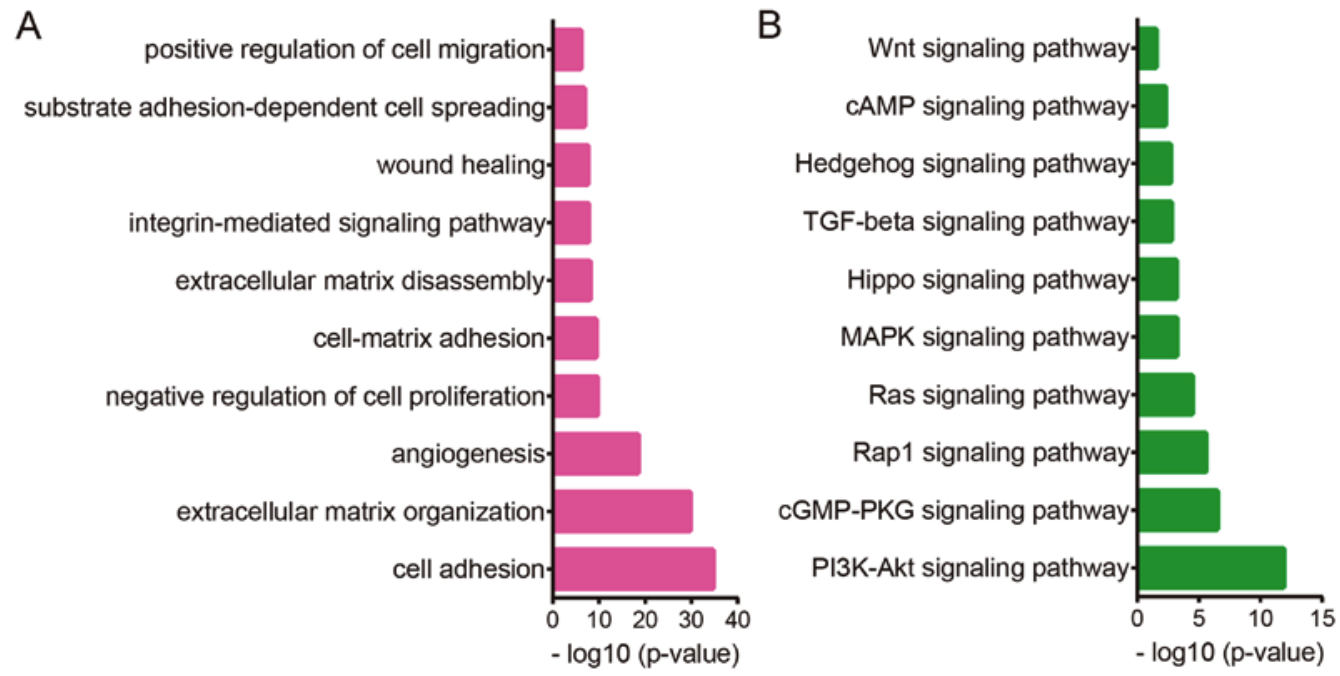

Figure 5. Bioinformatics analysis for COL8A1 in GC. (A) Biological processes analysis revealed the potential roles of COL8A1 in GC. (B) Kyoto Encyclopedia of Genes and Genomes pathways analysis revealed the potential roles of COL8A1 in GC. GC, gastric cancer.

In order to further validate this, Kaplan-Meier survival curves were used to analyze the association between COL8A1 levels and OS time in GC samples. The GSE15459, GSE29272, GSE51105, GSE62254, GSE15459 and GSE14210 datasets and the Kaplan-Meier Plotter database were analyzed. Similarly to TCGA analysis, patients with GC with a high COL8A1 expression level had a shorter OS time compared with those with low expression. Interestingly, high COL8A1 expression was associated with a shorter OS time in both human epidermal growth factor receptor 2 (HER2)-positive and negative GC samples (Fig. 4).

Bioinformatics analysis reveals the potential functions of COL8A1 in GC. The present study conducted co-expression 

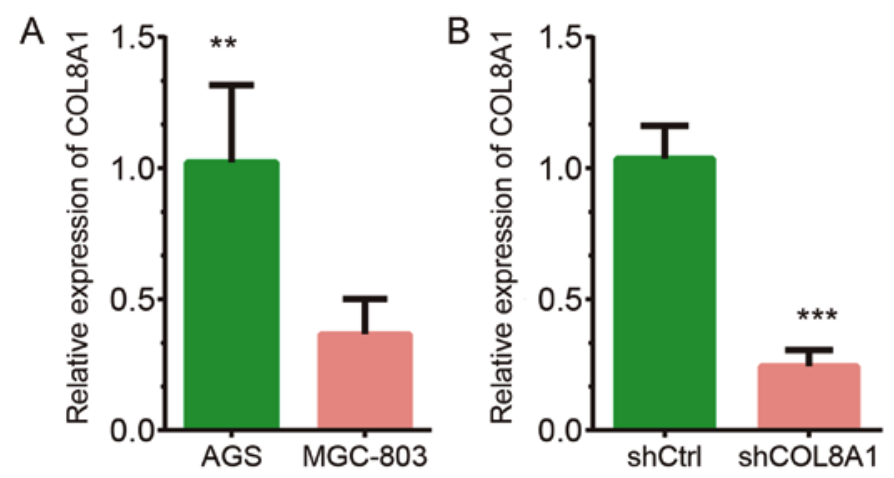

C

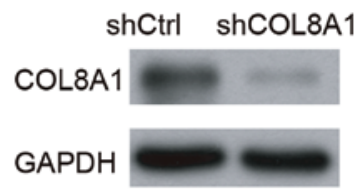

Figure 6. COL8A1 is effectively knocked down with lentivirus-mediated shCOL8A1 in GC AGS cells. (A) COL8A1 expressed in AGS and MGC-803 cells. (B) mRNA levels of COL8A1 were suppressed by shCOL8A1 in AGS cells. (C) Protein levels of COL8A1 were suppressed in AGS cells. All experiments were performed at least three times independently. ${ }^{* *} \mathrm{P}<0.01$ and ${ }^{* * * *} \mathrm{P}<0.001$ vs. shCtrl. sh, short hairpin RNA; GC, gastric cancer.
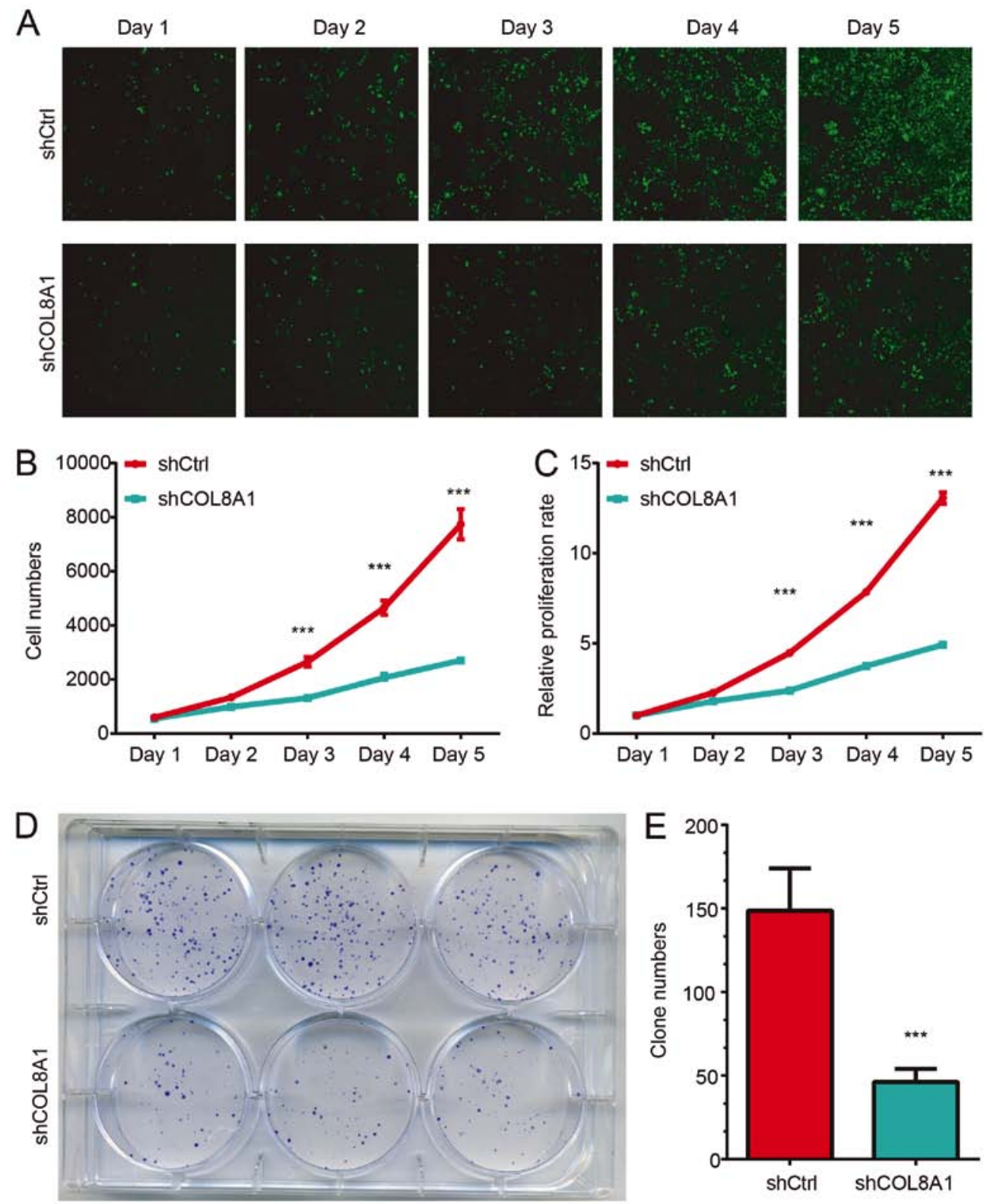

Figure 7. Inhibitory effects of COL8A1 knockdown on AGS cell growth. (A) Celigo cell count assay showed the AGS cell counts after transfection with shCtrl lentivirus and shCOL8A1 lentivirus on days 1,2,3,4 and 5. Magnification, x100. (B) Cell numbers of AGS cells expressing shCtrl lentivirus and shCOL8A1 lentivirus were determined using a Celigo Cell Counting application assay. (C) Cell proliferation fold of AGS cells expressing shCtrl lentivirus and shCOL8A1 lentivirus using Celigo Cell Counting application assay. (D) Representative microscopic images of colonies stained with crystal violet in AGS cells. (E) Statistical analysis of the number of colonies in AGS cells. All experiments were performed at least thrice and independently. ${ }^{* * *} \mathrm{P}<0.001 \mathrm{vs}$. shCtrl. NC, negative control; sh, short hairpin RNA; Ctrl, control. 
A
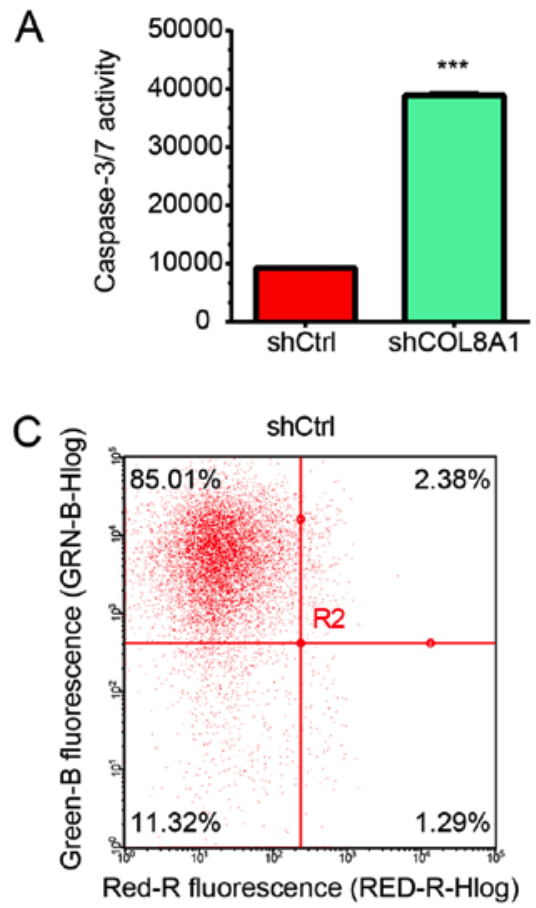

B

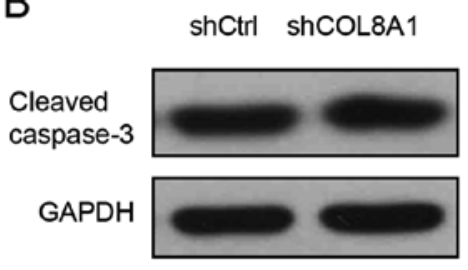

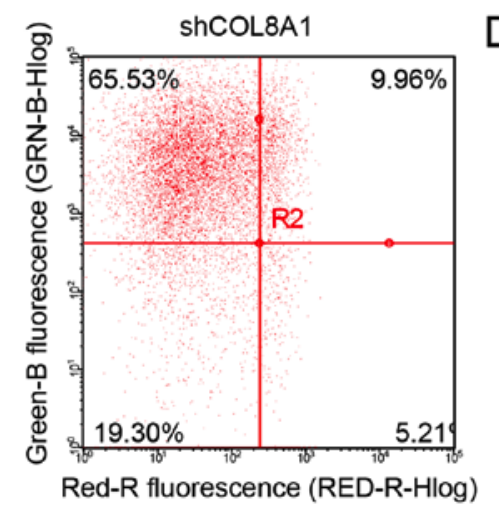

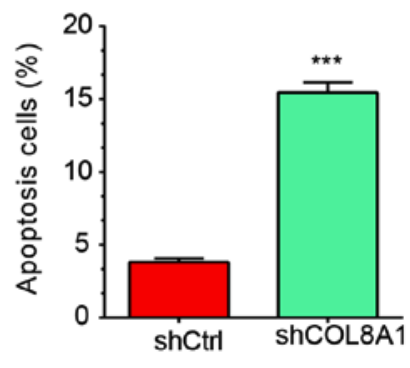

Figure 8. Depletion of COL8A1 induces apoptosis in RKO cells. (A) Caspase3 and caspase7 activity were significantly increased following COL8A1 knockdown in AGS cells. (B) Protein levels of cleaved-caspase3 were increased after COL8A1 knockdown in AGS cells in a western blotting assay. (C) Cell apoptosis induction was analyzed by flow cytometry. (D) Statistical analysis of the apoptosis induction following COL8A1 knockdown. All experiments were performed at least three times independently. ${ }^{* * * *} \mathrm{P}<0.001$ vs. shCtrl. sh, short hairpin RNA; Ctrl, control.

and bioinformatics analysis to reveal the potential roles of COL8A1 in GC. TCGA STAD dataset was used to perform co-expression analysis and a total of 1,461 genes with an absolute Pearson's correlation coefficient $>0.4$ were identified as the potential targets of COL8A1. Kyoto Encyclopedia of Genes and Genomes (KEGG) and Gene Ontology (GO) analyses using DAVID were subsequently performed.

KEGG analysis revealed that COL8A1 was significantly involved in regulating 'PI3K-Akt signaling', 'cGMP-PKG signaling', 'Rap1 signaling', 'Ras signaling', 'MAPK signaling', 'hippo signaling', 'TGF- $\beta$ signaling', 'hedgehog signaling', 'cAMP signaling' and 'Wnt signaling' (Fig. 5B). GO analysis demonstrated that COL8A1 was associated with the 'regulation of cell adhesion', 'extracellular matrix organization', 'angiogenesis', 'skeletal system development', 'regulation of cell proliferation', 'cell-matrix adhesion', 'extracellular matrix disassembly', 'wound healing', 'negative regulation of angiogenesis' and 'positive regulation of cell migration' (Fig. 5A). There results suggested that COL8A1 may participate in regulating GC cell proliferation and migration.

Knockdown of COL8Al expression in AGS cells. RT-qPCR results revealed that COL8A1 was highly expressed in AGS cells compared to MG-803 cells (Fig. 6A). A loss-of-function assay was subsequently performed to explore the functions of COL8A1 in GC. COL8A1 expression in AGS cells was knocked down using lentivirus-mediated gene transfection. As shown in Fig. 6B and C, both the mRNA and protein levels of COL8A1 were reduced in AGS cells following transfection with shCOL8A1 compared with shNC.
Silencing of COL8A1 suppresses AGS cell proliferation and colony formation. A Celigo ${ }^{\circledR}$ Cell Counting assay was used to detect AGS proliferation following COL8A1 knockdown. The results revealed that the proliferation rate of AGS cells transfected with shCOL8A1 was significantly inhibited compared with cells transfected with shNC. As indicated in Fig. 7A, the average cell number in the shCOL8A1-transfected group decreased by about $61 \%$ compared with the shNC-transfected group on day 5.

The colony formation assay revealed similar results to the cell proliferation assay. As shown in Fig. 7, shCOL8A1-transfected cells formed fewer and smaller colonies compared with the shNC-transfected group. Statistical analysis further confirmed that knockdown of COL8A1 significantly reduced the number of colonies (Fig. 7B-C; P<0.001). Collectively, these results suggested that knockdown of COL8A1 inhibited the proliferation of AGS cells.

COL8A1 knockdown promotes the apoptosis of GC cells. Bioinformatics analysis revealed that COL8A1 was involved in regulating apoptosis-related pathways. Therefore, the effect of COL8A1 knockdown on the apoptosis of AGS cells was investigated. As shown in Fig. 8A, the activity of caspase 3 and 7 was significantly increased following COL8A1 knockdown in AGS cells compared with the shNC group. Moreover, we detected the protein levels of cleaved caspase-3 using western blot assay. The results showed that the levels of cleaved caspase-3 were significantly up-regulated after COL8A1 knockdown in GC cells (Fig. 8B). These results suggested that COL8A1 knockdown significantly induces apoptosis of AGS cells (Fig. 8C). Furthermore, Annexin V-APC staining revealed that COL8A1 knockdown significantly promoted 
the apoptosis of AGS cells compared with the shNC group (Fig. 8D).

\section{Discussion}

COL8A1 is a collagen type VIII protein. Previous studies have demonstrated that COL8A1 is upregulated in several types of human cancer $(12,26,27)$. COL8A1 was found to be associated with tumor stage and prognosis in patients with bladder (12) and colon cancer (13). Furthermore, knockdown of COL8A1 inhibited the growth and invasion of hepatocellular carcinoma (14). Additionally, COL8A1 was reported to be involved in the regulation of GC progression $(26,28)$. To the best of our knowledge, the present study was the first to demonstrate that COL8A1 was upregulated in several types of cancer, including HNSC, KIRC, PRAD, CHOL, CESC, BRCA, READ, LUSC, COAD, ESCA, SKCM, BLCA, THCA, STAD and LIHC, and was associated with a shorter OS time. These results suggested that COL8A1 may serve as a potential oncogene in human cancer.

$\mathrm{GC}$ is one of the most common tumors of the digestive system and is associated with a poor prognosis $(29,30)$. Previous studies have investigated potential biomarkers for GC. Gastric cancer metastasis-associated long non-coding RNA was upregulated in patients with GC and was associated with metastasis (31). Upregulation of erythrocyte membrane protein band 4.1 like 5 was correlated with poor prognosis and a shorter survival time in patients with GC (32). However, despite the aforementioned advances, there is still an urgent need to identify effective biomarkers for GC. The present study analyzed a series of public databases, including TCGA and the Kaplan-Meier Plotter, to reveal that COL8A1 was upregulated in advanced GC samples compared with stage I GC samples. Moreover, the results demonstrated that patients with GC with high COL8A1 expression had a shorter OS time compared with those with low expression. Interestingly, a high expression of COL8A1 was associated with a shorter OS time in both HER2-positive and negative GC samples. These results suggested that COL8A1 may serve as a potential biomarker for GC.

The present study performed co-expression, GO and KEGG pathway analyses to explore the potential roles of COL8A1 in GC. The results revealed that COL8A1 was involved in regulating several proliferation and metastasis-related biological processes, including 'cell adhesion', 'regulation of cell proliferation', and 'positive regulation of cell migration'. KEGG pathways analysis showed that COL8A1 was associated with the regulation of the $\mathrm{PI} 3 \mathrm{~K}-\mathrm{Akt}$, mitogen-activated protein kinase (MAPK), hippo and transforming growth factor $\beta$ signaling pathways. Previous studies have demonstrated that the PI3K-Akt signaling played an important role in regulating GC cell cycle, proliferation, and chemotherapy resistance (33). Furthermore, the MAPK signaling pathway was associated with the regulation of GC metastasis (34). The present study performed a loss-of-function assay to validate the influence of COL8A1 on GC cell proliferation and migration. Collectively, the results suggested that COL8A1 serves as an oncogene in $\mathrm{GC}$ by promoting the proliferation and suppressing apoptosis of GC cells.

In conclusion, the present study revealed that COL8A1 was upregulated in several types of human cancer and was associated with a shorter OS time. Bioinformatics analysis revealed that COL8A1 was involved in regulating cell proliferation and metastasis. Experimental validation of COL8A1 expression demonstrated that silencing of COL8A1 significantly decreased GC cell proliferation in vitro. Therefore, COL8A1 may serve as a potential therapeutic target or prognostic biomarker for GC.

\section{Acknowledgements}

Not applicable.

\section{Funding}

No funding was received.

\section{Availability of data and materials}

The datasets used and/or analyzed during the current study are available from the corresponding author on reasonable request.

\section{Authors' contributions}

JZ and YC designed the study. YS, WG and PW collected and analyzed public data. JZ and LL conducted bioinformatics analysis. JZ, GC, ZC, GL and GZ performed the experiments. JZ and LZ performed the statistical analyses. All authors read and approved the final manuscript.

\section{Ethics approval and consent to participate}

Not applicable.

\section{Patient consent for publication}

Not applicable.

\section{Competing interests}

The authors declare that they have no competing interests.

\section{References}

1. Allison KH and Sledge GW: Heterogeneity and cancer. Oncology (Williston Park) 28: 772-778, 2014.

2. Hutter $\mathrm{C}$ and Zenklusen JC: The cancer genome atlas: Creating lasting value beyond its data. Cell 173: 283-285, 2018.

3. Tang Z, Li C, Kang B, Gao G, Li C and Zhang Z: GEPIA: A web server for cancer and normal gene expression profiling and interactive analyses. Nucleic Acids Res 45: W98-W102, 2017.

4. GTEx Consortium: Human genomics. The genotype-tissue expression (GTEx) pilot analysis: Multitissue gene regulation in humans. Science 348: 648-660, 2015.

5. Zhao B, Zhang J, Chen X, Xu H and Huang B: Mir-26b inhibits growth and resistance to paclitaxel chemotherapy by silencing the CDC6 gene in gastric cancer. Arch Med Sci 15: 498-503, 2019.

6. Wang W, Zhang Y, Liu M, Wang Y, Yang T, Li D, Ding F, Bai G and Li Q: TIMP2 is a poor prognostic factor and predicts metastatic biological behavior in gastric cancer. Sci Rep 8: 9629, 2018.

7. Li X, Wang Z, Tong H, Yan Y and Li S: Effects of COL8A1 on the proliferation of muscle-derived satellite cells. Cell Biol Int 42: 1132-1140, 2018. 
8. Plenz GA, Deng MC, Robenek H and Volker W: Vascular collagens: Spotlight on the role of type VIII collagen in atherogenesis. Atherosclerosis 166: 1-11, 2003.

9. Ooshima A and Muragaki Y: Collagen metabolism in atherogenesis. Ann NY Acad Sci 598: 582-584, 1990.

10. Aldave AJ, Bourla N, Yellore VS, Rayner SA, Khan MA, Salem AK and Sonmez B: Keratoconus is not associated with mutations in COL8A1 and COL8A2. Cornea 26: 963-965, 2007.

11. Aldave AJ, Rayner SA, Salem AK, Yoo GL, Kim BT, Saeedian M, Sonmez B and Yellore VS: No pathogenic mutations identified in the COL8A1 and COL8A2 genes in familial Fuchs corneal dystrophy. Invest Ophthalmol Vis Sci 47: 3787-3790, 2006.

12. Di Y, Chen D, Yu W and Yan L: Bladder cancer stage-associated hub genes revealed by WGCNA co-expression network analysis. Hereditas 156: 7, 2019.

13. Shang J, Wang F, Chen P, Wang X, Ding F, Liu S and Zhao Q: Co-expression network analysis identified COL8A1 is associated with the progression and prognosis in human colon adenocarcinoma. Dig Dis Sci 63: 1219-1228, 2018.

14. Zhao Y, Jia L, Mao X, Xu H, Wang B and Liu Y: siRNA-targeted COL8A1 inhibits proliferation, reduces invasion and enhances sensitivity to D-limonence treatment in hepatocarcinoma cells IUBMB Life 61: 74-79, 2009.

15. Subhash VV, Yeo MS, Wang L, Tan SH, Wong FY, Thuya WL, Tan WL, Peethala PC, Soe MY, Tan DSP, et al: Anti-tumor efficacy of Selinexor (KPT-330) in gastric cancer is dependent on nuclear accumulation of p53 tumor suppressor. Sci Rep 8: 12248 2018.

16. Li WQ, Hu N, Burton VH, Yang HH, Su H, Conway CM, Wang L, Wang C, Ding T, Xu Y, et al: PLCE1 mRNA and protein expression and survival of patients with esophageal squamous cell carcinoma and gastric adenocarcinoma. Cancer Epidemiol Biomarkers Prev 23: 1579-1588, 2014.

17. Brasacchio D, Busuttil RA, Noori T, Johnstone RW, Boussioutas A and Trapani JA: Down-regulation of a pro-apoptotic pathway regulated by PCAF/ADA3 in early stage gastric cancer. Cell Death Dis 9: 442, 2018.

18. Cristescu R, Lee J, Nebozhyn M, Kim KM, Ting JC, Wong SS, Liu J, Yue YG, Wang J, Yu K, et al: Molecular analysis of gastric cancer identifies subtypes associated with distinct clinical outcomes. Nat Med 21: 449-456, 2015.

19. Ooi CH, Ivanova T, Wu J, Lee M, Tan IB, Tao J, Ward L, Koo JH, Gopalakrishnan V, Zhu Y, et al: Oncogenic pathway combinations predict clinical prognosis in gastric cancer. PLoS Genet 5 : e1000676, 2009

20. Kim HK, Choi IJ, Kim CG, Kim HS, Oshima A, Yamada Y, Arao T, Nishio K, Michalowski A and Green JE: Three-gene predictor of clinical outcome for gastric cancer patients treated with chemotherapy. Pharmacogenomics J 12: 119-127, 2012.
21. Nagy Á, Lánczky A, Menyhárt O and Győrffy B: Validation of miRNA prognostic power in hepatocellular carcinoma using expression data of independent datasets. Sci Rep 8: 9227, 2018.

22. Zhao X, Hao S, Wang M, Xing D and Wang C: Knockdown of pseudogene DUXAP8 expression in glioma suppresses tumor cell proliferation. Oncol Lett 17: 3511-3516, 2019.

23. Peng C, Li X, Yu Y and Chen J: LncRNA GASL1 inhibits tumor growth in gastric carcinoma by inactivating the $\mathrm{Wnt} / \beta$ catenin signaling pathway. Exp Ther Med 17: 4039-4045, 2019.

24. Livak KJ and Schmittgen TD: Analysis of relative gene expression data using real-time quantitative PCR and the 2(-Delta Delta C(T)) method. Methods 25: 402-408, 2001.

25. Jin H, Zhang Q, Zhang S, Liu H, Man Z and Wang Y: Effects of claudin-1 downregulation on the physiological processes of gallbladder cancer SGC996 cells. Oncol Lett 17: 1688-1694, 2019.

26. Liu X, Wu J, Zhang D, Bing Z, Tian J, Ni M, Zhang X, Meng Z and Liu S: Identification of potential key genes associated with the pathogenesis and prognosis of gastric cancer based on integrated bioinformatics analysis. Front Genet 9: 265, 2018.

27. Wang Z, Chen G, Wang Q, Lu W and Xu M: Identification and validation of a prognostic 9-genes expression signature for gastric cancer. Oncotarget 8: 73826-73836, 2017.

28. Chen J, Wang X, Hu B, He Y, Qian X and Wang W: Candidate genes in gastric cancer identified by constructing a weighted gene co-expression network. Peer J 6: e4692, 2018.

29. Bray F, Ferlay J, Soerjomataram I, Siegel RL, Torre LA and Jemal A: Global cancer statistics 2018: GLOBOCAN estimates of incidence and mortality worldwide for 36 cancers in 185 countries. CA Cancer J Clin 68: 394-424, 2018.

30. Chen W, Zheng R, Baade PD, Zhang S, Zeng H, Bray F, Jemal A, Yu XQ and He J: Cancer statistics in China, 2015. CA Cancer J Clin 66: 115-132, 2016.

31. Zhuo W, Liu Y, Li S, Guo D, Sun Q, Jin J, Rao X, Li M, Sun M, Jiang $\mathrm{M}$, et al: Long noncoding RNA GMAN, up-regulated in gastric cancer tissues, is associated with metastasis in patients and promotes translation of ephrin a1 by competitively binding GMAN-AS. Gastroenterology 156: 676-691, 2019.

32. Jeong MH, Park SY, Lee SH, Seo J, Yoo JY, Park SH, Kim MJ, Lee S, Jang S, Choi HK, et al: EPB41L5 mediates TGF $\beta$-induced metastasis of gastric cancer. Clin Cancer Res 25: 3617-3629, 2019.

33. Almhanna K, Strosberg J and Malafa M: Targeting AKT protein kinase in gastric cancer. Anticancer Res 31: 4387-4392, 2011.

34. Yang $M$ and Huang CZ: Mitogen-activated protein kinase signaling pathway and invasion and metastasis of gastric cancer. World J Gastroenterol 21: 11673-11679, 2015.

This work is licensed under a Creative Commons Attribution-NonCommercial-NoDerivatives 4.0 International (CC BY-NC-ND 4.0) License. 